\title{
Comet 67P/Churyumov-Gerasimenko at a large heliocentric distance ${ }^{\star}$
}

\author{
C. Tubiana ${ }^{1}$, L. Barrera ${ }^{2}$, M. Drahus ${ }^{1}$, and H. Boehnhardt ${ }^{1}$ \\ 1 Max Planck Institute fuer Sonnensystemforschung, Max-Planck-Str. 2, 37191 Katlenburg-Lindau, Germany \\ e-mail: [tubiana; boehnhardt;drahus] @mps .mpg.de \\ 2 Universidad Metropolitana de Ciencias de la Educación, Santiago, Chile \\ e-mail: lbarrera@umce.cl
}

Received 4 October 2007 / Accepted 15 July 2008

\section{ABSTRACT}

\begin{abstract}
Aims. The Jupiter family comet 67P/Churyumov-Gerasimenko (67P/C-G) is the target comet of ESA's ROSETTA mission. A detailed portrait of this comet has been drawn from observations around perihelion, but what needs to be enhanced is the description of the comet's behaviour at large heliocentric distance. It is not only important for planning the rendezvous of the ROSETTA spacecraft with the comet, but will also provide valuable information on basic physical properties of the nucleus and the possible distant activity of JFCs.

Methods. We performed broad-band imaging and longslit spectroscopy of 67P/C-G in the visible wavelength range in June 2004, May 2006, and August 2006, when the comet was at the heliocentric distance of $r \geq 4.9$ AU. The observations were performed at the ESO VLT with the FORS2 instrument.

Results. The comet appears point-like at all observing epochs, indicating that no significant coma is present around the nucleus. From our $67 \mathrm{P} / \mathrm{C}-\mathrm{G}$ images, we determined a synodic rotational period of the nucleus of $12.7047 \pm 0.0011 \mathrm{~h}$ and a linear phase function coefficient $\beta=0.076 \pm 0.003 \mathrm{mag} /{ }^{\circ}$. We estimated the large-to-small axis ratio $a / b \geq 1.45 \pm 0.09$ and, assuming an albedo of 0.04 , an effective nucleus radius of $2.38 \pm 0.04 \mathrm{~km}$, which corresponds to the mean absolute magnitude $H=15.35 \pm 0.04$ mag. No colour variation with the rotational phase is found, based on broadband colour indices and reflectance spectra of the nucleus. A trail-like structure of heavy grains is clearly detected in June 2004, displaying a surface brightness of $\sim 28 \mathrm{mag} /{ }^{\prime \prime} 2$ in $R$ the filter. For the first time we also determined visible colours of the trail-like structure: $(V-R)=0.95 \pm 0.14 \mathrm{mag} /{ }^{\prime \prime 2}$ and $(R-I)=0.39 \pm 0.14 \mathrm{mag} /{ }^{\prime \prime 2}$. A trail-like structure is also barely visible in the May 2006 images, but a quantitative study was not possible.
\end{abstract}

Key words. comets: general - comets: individual: comet 67P/Churyumov-Gerasimenko - methods: observational techniques: photometric - techniques: spectroscopic - solar system: general

\section{Introduction}

After the success of the Giotto mission to comet 1P/Halley, the European Space Agency (ESA) approved a new space mission in the early nineties with a comet as its main target. ROSETTA will rendezvous with the Jupiter family comet $67 \mathrm{P} /$ ChuryumovGerasimenko (67P/C-G) in 2014. It will be the first spacecraft to orbit a comet's nucleus and to land on it. What makes this mission exceptional is that the spacecraft will follow the comet in its journey towards the Sun, examining how a frozen comet is transformed by solar heating and space weathering. In 2003, due to a delay in the launch of the ROSETTA spacecraft, 67P/C-G was chosen on the basis of orbital considerations as the most suitable alternative to the original target, 46P/Wirtanen, even though very little was known at that time about this comet and the physical properties of its nucleus. Since then, observational campaigns and theoretical investigations were performed to establish a detailed portrait of $67 \mathrm{P} / \mathrm{C}-\mathrm{G}$ in preparation for the rendezvous with the spacecraft. Due to repeated close encounters with Jupiter, the orbital evolution of $67 \mathrm{P} / \mathrm{C}-\mathrm{G}$ is chaotic. The last encounter in February 1959 produced a drastic change in the orbit of

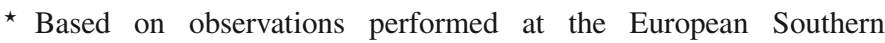
Observatory, Chile (ESO Programmes 073.C-0346, 077.C-0609 \& 277.C-5038)
67P/C-G and may have triggered its activity, which most likely led to its discovery in 1969 (Lamy et al. 2007).

The most recent results for physical properties of $67 \mathrm{P} / \mathrm{C}-\mathrm{G}$ have been collected by various scientists and are summarised by Lamy et al. (2007) for nucleus and gas, Agarwal et al. (2007) for dust and Hansen et al. (2007) for plasma. Thanks to spaceand ground-based observations performed around perihelion in different wavelength regions, a detailed portrait of the comet has been drawn. What needs to be enhanced in this picture of the ROSETTA target is a profound description of the comet's behaviour at large heliocentric distances. A first characterisation of the physical properties of $67 \mathrm{P} / \mathrm{C}-\mathrm{G}$ far away from the Sun has been obtained by Lowry et al. (2006). Here, we present the results of visible imaging and spectroscopy of $67 \mathrm{P} / \mathrm{C}-\mathrm{G}$ observed at the ESO Very Large Telescope (VLT) in June 2004, May 2006, and August 2006, when the comet was at the heliocentric distance of $r \geq 4.9$ AU. The main aim of our observations was to investigate the possible presence of activity in the aphelion arc. Since no activity was detected, we focused our analysis mainly on the characterisation of the nucleus of $67 \mathrm{P} / \mathrm{C}-\mathrm{G}$ and its dust trail. Thus, our observations of $67 \mathrm{P} / \mathrm{C}-\mathrm{G}$ are not only important for good planning of the rendezvous of the ROSETTA spacecraft with the comet, but also provide valuable information on the basic physical properties of the nucleus of JFCs. 
Table 1. Details of the observations of $67 \mathrm{P} / \mathrm{C}-\mathrm{G}$.

\begin{tabular}{c|c|c|c|c|c|c|c}
\hline \hline Start time [UT] & End time [UT] & $r[\mathrm{AU}]$ & $\Delta[\mathrm{AU}]$ & $\alpha\left[^{\circ}\right]$ & $G L\left[^{\circ}\right]$ & Sky cond. & Observ. type \\
\hline $16.06 .0423: 20$ & $17.06 .0402: 17$ & 4.89 & 4.29 & 10.3 & 52.6 & PH & IM \\
\hline $26.05 .0601: 00$ & $26.05 .0609: 42$ & 5.62 & 4.61 & 1.3 & 14.7 & CL & IM \& SP \\
$31.05 .0600: 28$ & $31.05 .0609: 33$ & 5.61 & 4.60 & 0.6 & 15.3 & CL & IM \& SP \\
$01.06 .0600: 17$ & $01.06 .0609: 46$ & 5.61 & 4.60 & 0.5 & 15.5 & CL & IM \& SP \\
\hline $18.08 .0601: 42$ & $18.08 .0602: 03$ & 5.51 & 5.27 & 10.5 & 21.3 & CL & IM \\
$19.08 .0601: 29$ & $19.08 .0601: 48$ & 5.51 & 5.28 & 10.5 & 21.2 & CL & IM \\
$23.08 .0602: 32$ & $23.08 .0602: 52$ & 5.50 & 5.34 & 10.6 & 21.1 & CL & IM \\
$23.08 .06 ~ 23: 30$ & $23.08 .0623: 53$ & 5.50 & 5.35 & 10.6 & 21.1 & CL & IM \\
\hline
\end{tabular}

Explanation: date and start \& end time of the observations; $r$ and $\Delta$ are the heliocentric and geocentric distances; $\alpha$ is the phase angle and GL the galactic latitude of the comet. The average sky conditions during the night are reported $(\mathrm{PH}=$ photometric, $\mathrm{CL}=$ clear $)$ as well as the type of observations performed (IM = imaging, $\mathrm{SP}=$ spectroscopy).

\section{Observations and data reduction}

We observed $67 \mathrm{P} / \mathrm{C}-\mathrm{G}$ in the visible wavelength range, on June 16, 2004, May 25, 30, and 31, 2006 and August 17, 18, 22, and 23, 2006 with the FORS2 instrument at the $8.2 \mathrm{~m}$ Very Large Telescope UT1 (named Antu) in Cerro Paranal (Chile). FORS2 (for a description of the instrument, see http: //www. eso . org/ instruments/fors/index.html) is one of the two visual and near UV FOcal Reducer and low dispersion Spectrographs for the VLT of the European Southern Observatory (ESO). Its detector consists of a mosaic of two $2 \mathrm{k} \times 4 \mathrm{k}$ MIT CCDs $(15 \mu \mathrm{m}$ pixels). In the standard resolution mode (which we used for the observations), the image scale in the default readout mode ( $2 \times 2$ pixel binning) is $0.25^{\prime \prime} /$ pix, providing a field of view of $6.8^{\prime} \times 6.8^{\prime}$. The observations were performed with the telescope tracking at the proper motion rate of the comet. The observational circumstances in the observation nights are summarised in Table 1.

The aim of each of the observing runs was different, hence also the strategy applied for the observations, as discussed in the subsequent paragraphs.

\subsection{Observations in June 2004}

The main purpose of the observing run was to detect and characterise the dust trail. Thus, three hours of observations were granted on June 16, 2004, when the comet was at a heliocentric distance of 4.9 AU, moving towards aphelion. The observation consisted of three consecutive series of VRI broadband images, one series per filter. The dataset is composed of a total of 38 images: 18 in $I$ Bessell filter $\left(\lambda_{\text {central, } \mathrm{I}}=7970 \AA\right), 10$ in $V$ Bessell filter $\left(\lambda_{\text {central, } \mathrm{V}}=5450 \AA\right)$, and 10 in the $R$ special filter $\left(\lambda_{\text {central, } \mathrm{R}}=6380 \AA\right)$. To ensure a good signal-to-noise ratio $(S / N \sim 50$ was anticipated $)$, we used an exposure time of $300 \mathrm{~s}$ for the $V$ and $R$ filter images. To avoid saturation of the CCD by sky background light, the $I$ filter images were split into two exposures of $150 \mathrm{~s}$ each. The images were reduced using standard techniques of bias subtraction, flat field correction (using a super-flat field created from the night exposures of the comet), exposure time normalisation, and sky background subtraction. To flux-calibrate the images, we observed a standard star field (PG1323-086) in the same instrument configuration as for the comet frames. From the standard star field, we determined the zero point, adopting colour terms, and extinction coefficients as provided by ESO for the period when the observations were carried out (http://archive.eso.org/bin/qc1_ cgi ?action=qc1_browse_instrume $-\backslash$ \&instrume=FORS2).

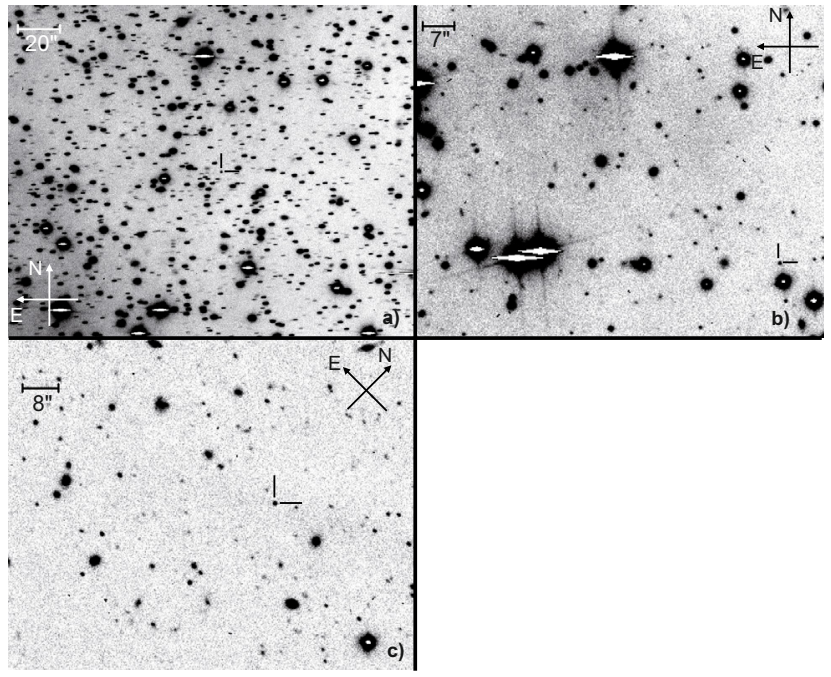

Fig. 1. $R$ filter images of 67P/C-G taken in May 2006 (panel a)), August 2006 (panel b)), and June 2004 (panel c)). The position of the comet in the frames is marked and the orientation of each frame is shown. Each panel shows only part of the original image. The images illustrate the crowded fields at low galactic latitudes (panels a) and b)), which makes accurate photometry of the comet a difficult task. The uneven diffuse background is due to galactic clouds.

\subsection{Observations in May 2006}

In May 2006 we observed 67P/C-G to characterise physical properties of its nucleus. Four full nights of observations were granted in order to fulfill the following goals:

1. assessment of the nucleus activity around aphelion and, if no activity is detected; then

2. accurate determination of the nucleus size and of the light curve over the rotation phase for a refinement of the rotational period of $12.41 \pm 0.41 \mathrm{~h}$ (Lamy et al. 2006) and $12.72 \pm 0.05 \mathrm{~h}$ (Lowry et al. 2006) and to achieve a firm reference for later studies of changes in the rotational period, due to nuclear activity, and light curve shape along the orbit due to changing viewing geometry;

3. measurement of possible VRI colour variations with rotational phase;

4. detection of potential minerals and absorptive materials on the nuclear surface through reflectance spectroscopy.

Due to bad weather conditions, one night was lost and the observations were performed on May 25, 30, and 31, 2006 (see Table 1). The comet was at the heliocentric distance of about 5.6 AU, post-aphelion, and at a galactic latitude of about $15^{\circ}$, thus in front of a star-rich background (see Fig. 1, panel a). 
The expected nucleus magnitude (estimated from previous measurements) was around 23 mag. Due to its motion, the comet passed close by or even across a number of background objects, making the photometric measurements difficult and in some cases even impossible. Thus, when a background star was contaminating the comet image or the sky background around, we discarded the image for photometric measurements. At the time of the observations, $67 \mathrm{P} / \mathrm{C}-\mathrm{G}$ was at phase angle between $0.5^{\circ}$ and $1.3^{\circ}$, an interesting range for assessing the opposition effect of the comet.

Photometry. A total of 197 broadband images of 67P/C-G were taken during the three nights of observations: 165 in the $R$ special filter $\left(\lambda_{\text {central, } \mathrm{R}}=6380 \AA\right), 8$ in the $V$ Bessell filter $\left(\lambda_{\text {central, } \mathrm{V}}=\right.$ $5450 \AA)$, and 24 in the $I$ Bessell filter $\left(\lambda_{\text {central, }, ~}=7970 \AA\right)$. The exposure time used were the same as in June 2004. Bias, flat fields, and standard stars were taken each night. Standard techniques of image reduction were applied, as described in Sect. 2.1. For the flux calibration, we determined the photometric coefficients (zero points, extinction coefficients, and colour terms) using the various standard star fields observed at different airmasses. We determined a unique photometric solution for the three nights of observation.

Spectroscopy. Spectra of the comet were obtained using the low-resolution grism 150I+27 $(\Delta \lambda=3500-11000 \AA$, dispersion $=3.45 \AA /$ pix). On May 25, 30, and 31, we used a $1.3^{\prime \prime}$ slit. The slit of the spectrograph was placed parallel to the direction of proper motion of the comet. Unfortunately, the spectrum observed on May 30 was too weak so it could not be used for analysis. For calibration purposes, spectra of solar analogue stars were taken at similar airmasses and with the instrument in the same configuration as for the comet's spectra . Each measurement of $67 \mathrm{P} / \mathrm{C}-\mathrm{G}$ was split into two exposures at two different slit positions A and B, 20" apart. By subtracting spectrum A and $\mathrm{B}$ from each other, a very accurate background subtraction was achieved. In order to increase the signal-to-noise ratio, the positive spectra resulting from $\mathrm{A}-\mathrm{B}$ and $\mathrm{B}-\mathrm{A}$ subtractions were aligned and summed-up. Standard methods for spectroscopy reduction and extraction were applied (bias subtraction, flat fielding using lamp flats, wavelength calibration through arclamp spectra, exposure time normalisation; extinction correction was not applied because not needed). To remove the solar spectrum from the comet ones, we divided the comet spectra by the average of the solar analogue spectra obtained with the same slit at similar airmasses. Finally, the comet spectra were normalised to unity at $6380 \AA$ (central wavelength of the $R$ filter).

\subsection{Observations in August 2006}

We performed $R$ filter imaging of $67 \mathrm{P} / \mathrm{C}-\mathrm{G}$ in parallel with scheduled Spitzer observations of the comet. We obtained 3 images of the comet per epoch, for a total of 4 epochs on August 17, 18,22 , and 23, when the comet was at about 5.5 AU from the Sun (post aphelion) and at a phase angle slightly more than $10^{\circ}$. To have a reasonably good signal-to-noise ratio, the exposure time of each image was set to $350 \mathrm{~s}$. Bias, flat fields, and photometric standards were taken each night. The images were reduced as described in Sect. 2.1. For the flux calibration, we determined the zero point using the standard star field PG1633+099, assuming the extinction coefficient and colour term as determined from the May 2006 measurements. The comet was still at low galactic latitude, thus the field of view was as crowded as during the May 2006 observations (see Fig. 1, panel b). Since the comet was always very close to bright stars, we subtracted rebinned images of different nights (centred on the same star) in order to remove the star background from the vicinity of the comet.

\subsection{Comet photometry}

Using normal aperture photometry, we measured the apparent magnitude of the comet $m(r, \Delta, \alpha)$ in each frame. From that we calculated the reduced magnitudes $m(1,1, \alpha)$ normalised to Sun and Earth distances equal to $1 \mathrm{AU}$, which are listed in Table 5. The observing time is corrected for the travel time of light to the comet. For the photometric error estimation, we considered the error on the aperture measurement, the error of the flux calibration coefficients, and errors due to the "non-flatness" of the flat field used. The final error on the apparent, reduced, and absolute magnitudes was obtained with the Gaussian error propagation. For the May and August 2006 datasets, we adopt a safe overall value of the photometric errors of $0.05 \mathrm{mag}$, although minor deviations exist for individual measurements (with smaller error estimates).

\section{Results on the cometary nucleus}

\subsection{Activity level at large distance (coma)}

We investigated the presence of coma around the nucleus of 67P/C-G in June 2004, May 2006, and August 2006. For all datasets we applied two methods:

1. We compared the point spread function (PSF) of the comet with the one of a neighbouring star following the approach described in Boehnhardt et al. (2002). Apart from minor offsets in the normalised peak magnitude (which is due to the statistical scatter of the brightest pixel position in the image centre) the profiles of the comet and star images are identical, indicating that the comet PSF is indistinguishable from that of a point source (Fig. 2); therefore, we conclude that no coma activity is detectable around the nucleus. Weak coma activity would first be detected by deviations of the comet PSF from that of the star in the inflection region, just before the profile becomes background-dominated.

2. We co-add images centred on the comet and searched for diffuse structures around the nucleus in the co-added image. In addition, we applied a smoothing of the co-added images to increase the $S / N$ ratio. No coma structure is visible in either the co-added images and the smoothed ones.

In conclusion, for each observing epoch, 67P/C-G appears like a point source, indicating that no coma was evident around the nucleus. We estimated that the limiting detectable brightness in the co-added image is $29.8 \mathrm{mag}$.

\subsection{Rotational period and phase function of the nucleus}

The nucleus of comet $67 \mathrm{P} / \mathrm{C}-\mathrm{G}$, since it is inactive during our observations and presumably has an irregular shape, should exhibit periodic variations in brightness, stimulated by its rotation. This is because the nucleus changes its projected cross-section over the rotational cycle, thus its surface reflects different amounts of light. Assuming shape-dominated variability, the rotational phase profile should feature two main peaks of brightness separated by approximately half of the rotational phase. However, the actual light curve may be quite irregular due to possible and irregular albedo and shape variations over the nucleus surface. 

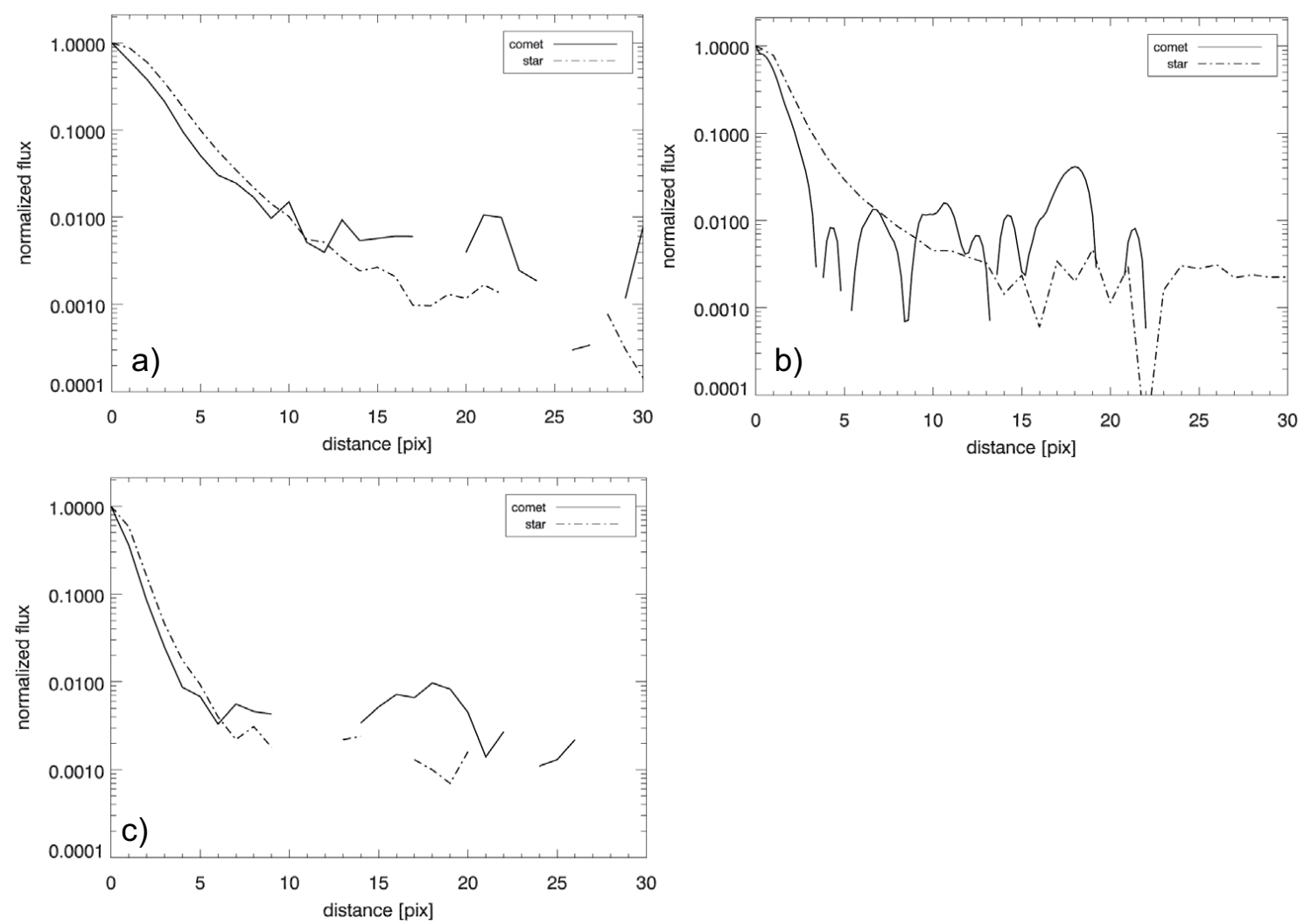

Fig. 2. Comparison between the profile of the comet (solid line) and the one of a neighbouring star (dashed line). The normalised flux is plotted versus the distance (in pixels) from the centre of the objects. Panel a): May 2006 observation, panel b): August 2006 observation, panel c): June 2004 observation.

Datasets. Although three datasets are available to us (June 2004, May 2006, and August 2006), to determine the rotation period and of the phase function coefficient of the comet, we restrict the analysis to the May 2006 and August 2006 datasets only, in order to ensure stability of the observing geometry.

Following the approach of Drahus \& Waniak (2006), who adopted the routine by Michałowski (1988), we calculated that the maximum phase shift induced by changes of the geometry of the comet between the two runs in 2006, is less than 0.01 at a $95 \%$ confidence level, i.e. negligibly small. This suggests negligible changes in the mean magnitude, amplitude, and specific shape of the phase profile, meaning that we can analyse the data from both epochs all together. Moreover, this also ensures that the rotational periodicity, though formally of a synodic type, is practically indistinguishable from the sidereal rotational period of the nucleus. In contrast, the maximum phase shift calculated for the 2004 and 2006 campaigns, due to the longer time difference between the two observing runs, is equal to 0.27 at a $95 \%$ confidence level. Changes in the viewing aspect of the nucleus that occurred during this time interval most likely produce considerable changes in the observed phase profile. Therefore, we do not consider the June 2004 observations for period analysis.

As input data we use the reduced magnitude $m(1,1, \alpha)$ as described in Sect. 2.4. The final dataset (May + August 2006) consists of 107 datapoints, 95 from May and 12 from August 2006.

Methods. When performing the periodicity analysis, we generally followed the approach of Drahus \& Waniak (2006). Since $67 \mathrm{P} / \mathrm{C}-\mathrm{G}$ was inactive over the time interval considered here, we chose the phase function coefficient as a second free parameter to be estimated simultaneously with the rotational period. Two different methods were used as searching kernels, i.e. the phase dispersion minimisation (hereafter PDM), originally proposed by Stellingwerf (1978), and the $\chi^{2}$ minimisation using a sum of harmonics fitted to the observations (hereafter $\chi^{2}$ minimisation). Both methods return the quantity $\Theta$ as a function of the rotational frequency $f$, whose minima indicate possible periodicities present in the light curve. The working parameters of the PDM algorithm are the number of bins $N_{\mathrm{b}}$ and the number of covers $N_{\mathrm{c}}$. The single parameter of the $\chi^{2}$ minimisation method is the number of frequencies $N_{\mathrm{f}}$, i.e. the number of harmonics including the base frequency that are used for fitting. Although both methods, as implemented by Drahus \& Waniak (2006), account for individual weights of the data points, we did not take advantage of this property for the rotational period and phase function coefficient estimation for $67 \mathrm{P} / \mathrm{C}-\mathrm{G}$, as the errors of our nucleus photometry were found to be rather uniform (0.05 mag, see Sect. 2, last paragraph).

The errors in the frequency (or periodicity) and in the linear phase function coefficient were retrieved through a Monte Carlo method:

1. Using the $\chi^{2}$ minimisation method, a smooth curve is calculated for the individual moments of the observations. (Here the $\chi^{2}$ minimisation is used for two different purposes: as a method for periodicity search and in order to get a noiseless representation of the phase profile.)

2. The smooth profile is modified by "data noise" calculated individually for each observing point, producing a set of simulated light curves. We assumed a Gaussian noise distribution, with its variance given by the square of the error bar.

3. We repeat the procedure for 1000 noise realisations and each of the simulated light curves is then analysed with the PDM and the $\chi^{2}$ minimisation methods. This provides 1000 frequencies and phase function coefficients for each method. 

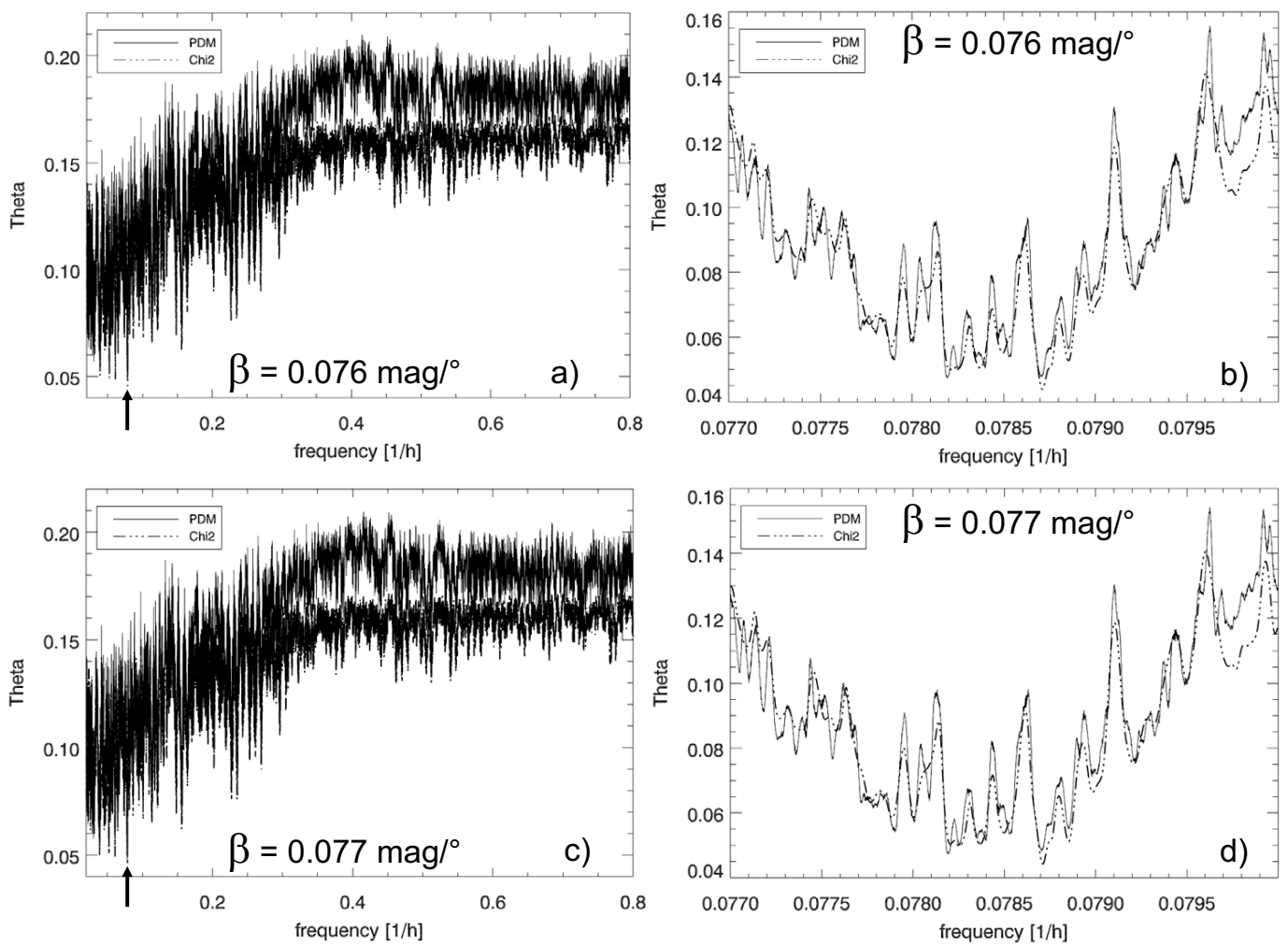

Fig. 3. Cross-cut through the $\Theta$ periodograms for the whole range of frequencies scrutinised (panels a)) and c)) for fixed values of the linear phase coefficient $\beta$. The regions of the global minima are marked by a black arrow and a 50-times zoom of these regions is plotted on the right hand side of the figure (panels b) and $\mathbf{d}$ )). The very low values of $\Theta$ result from the normalisation of this parameter using the datapoints, which were unphased and uncorrected for the phase angle dependence.

As errors on the frequency and on the phase function coefficient, we adopted the standard deviations of these quantities, resulting from each set of the 1000 solutions. This approach naturally converts the observing uncertainties into the errors of the quantities determined.

Results. We first consider the linear phase function. We scrutinised the frequency $f$ ranging from 0.02 to $0.8 \mathrm{~h}^{-1}\left(T_{\text {rot }}=\right.$ $1.25-50 \mathrm{~h}$ ) and the linear phase coefficient $\beta$ ranging from 0 to $0.2 \mathrm{mag} /{ }^{\circ}$. The PDM algorithm was used with $N_{\mathrm{b}}=25$ and $N_{\mathrm{c}}=5$, whereas the $\chi^{2}$ minimisation method was run for $N_{\mathrm{f}}=5$. We checked that, if working with noticeably lower values of these parameters, the methods would not return the whole set of existing solutions, whereas for much higher values they did not provide any additional solutions and were at the same time also less stable due to the limited number of measurements available.

Both the PDM and the $\chi^{2}$ minimisation methods show only one clear band of solutions (i.e., characterised by considerably deeper minima than any others), which is located around $f=0.078-0.079 \mathrm{~h}^{-1}$ and $\beta=0.07-0.09 \mathrm{mag} /{ }^{\circ}$ (Fig. 3). For PDM, the global minimum of $\Theta$ was found at $f_{1}=0.078193 \mathrm{~h}^{-1}$ $\left(T_{\text {rot }, 1}=12.7889 \mathrm{~h}\right)$ and $\beta_{1}=0.077 \mathrm{mag} /{ }^{\circ}$, whereas the $\chi^{2}$ minimisation method returned $f_{2}=0.078711 \mathrm{~h}^{-1}\left(T_{\mathrm{rot}, 2}=\right.$ $12.7047 \mathrm{~h}$ ) and $\beta_{2}=0.076 \mathrm{mag} /{ }^{\circ}$. The phase profiles corresponding to these rotational periods are plotted in Fig. 4 . The other potential solutions at lower frequencies (see Fig. 3) offer unrealistic rotational phase profiles.

The periodograms (Fig. 3) clearly show that the band is very complex, featuring several individual solutions. However, the global minimum given by one method (either PDM or $\chi^{2}$ minimisation) is detected as the second deepest minimum by the other one (either $\chi^{2}$ minimisation or PDM). This confirms the robustness of the minima found. The difference in frequency between the absolute minima from the two methods is equivalent to the uncertainty of one full rotational cycle between the May and August observing runs; thus for each of the solutions, the data are phased very similarly.

As mentioned before, other solutions are also visible in between $f_{1}$ and $f_{2}$. They are characterised by different rotational phases of the August data and look less realistic (which is reflected by higher values of $\Theta$ ), but they cannot be completely excluded. Therefore, $f_{1}$ and $f_{2}$ could also be considered as the extremes of a broader range of possible, although less likely, rotational frequencies.

The two solutions for the rotational frequency have practically the same value of the linear phase function coefficient $\beta$, which is mainly controlled by the large phase angle difference between the May and August observations (see Table 1). These best-fit $\beta$ values $\left(0.077-0.076 \mathrm{mag} /{ }^{\circ}\right)$ correspond to a very steep brightness dependence on phase angle. For cometary nuclei, a shallower dependence is usually suggested, with typical values of $\beta$ around $0.04 \pm 0.02 \mathrm{mag} /{ }^{\circ}$ (Lamy et al. 2004). However, a steeper phase function is measured, for instance, for comet 2P/Encke (see Fernandez et al. 2000).

For each of the considered solutions, the uncertainties of the rotational frequency (and thus the rotational period) and the linear phase function coefficient were estimated using 1000 realisations of Gaussian noise superimposed on smooth representations of the corresponding phase profiles (cf. the description in paragraph "Methods", in this section). The smooth fit was calculated with $N_{\mathrm{f}}=8$, which offers the most realistic curve, such that the dispersion of the data points about the fit was closest to the rms error of the measured photometry. For both solutions the derived 

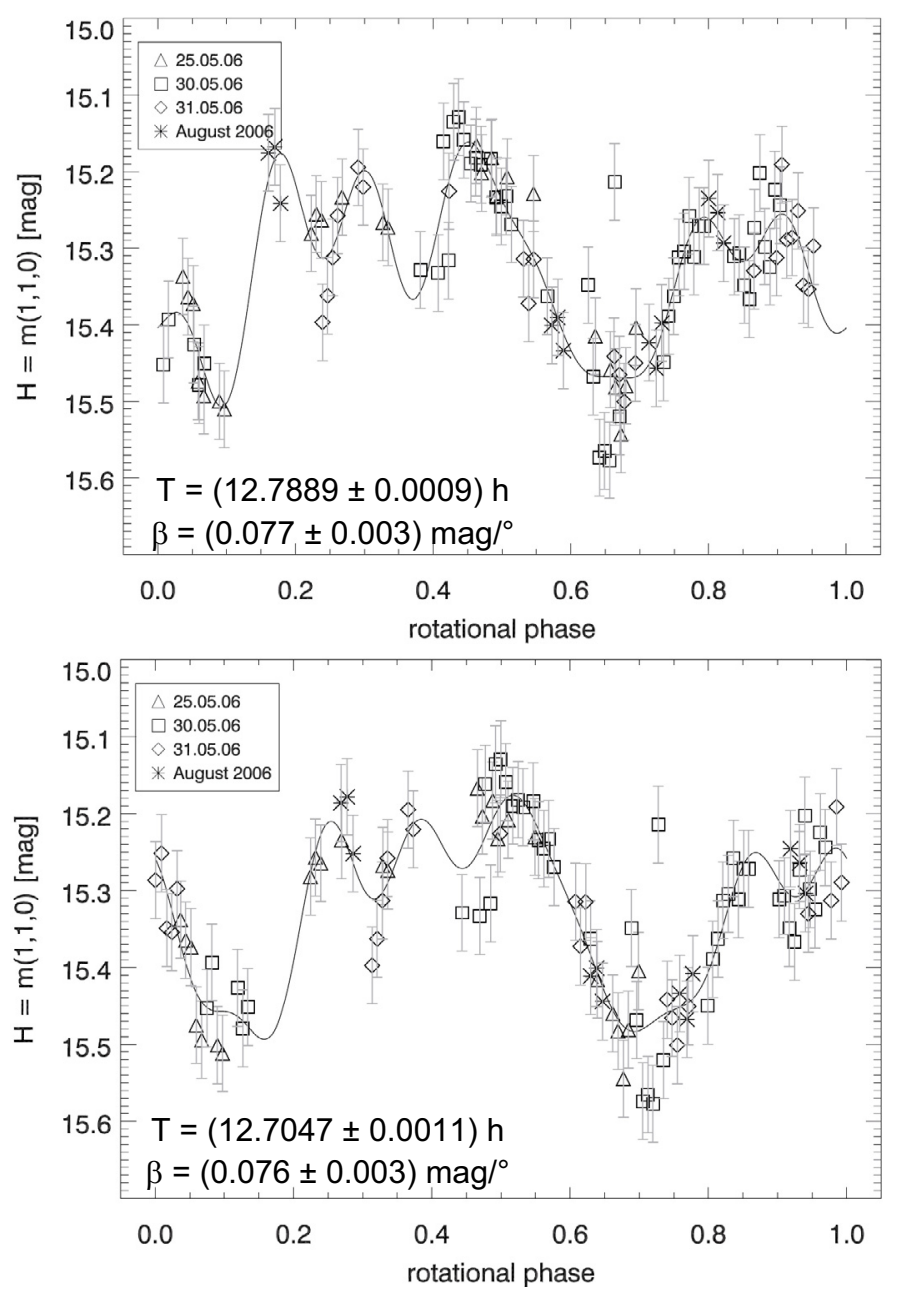

Fig. 4. Rotational phase profiles of both solutions for the fit assuming a linear phase function. The smooth curves were calculated using the $\chi^{2}$ minimisation method with $N_{\mathrm{f}}=8$. They were used as reference profiles for calculating the errors of the solutions.

Table 2. Best solutions for rotational periods and linear phase coefficients of 67P/C-G using the May and August 2006 photometry of the nucleus.

\begin{tabular}{ccc}
\hline \hline & $T_{\text {rot }}[\mathrm{h}]$ & $\beta\left[\mathrm{mag} /{ }^{\circ}\right]$ \\
\hline Solution 1 & $12.7889 \pm 0.0009$ & $0.077 \pm 0.003$ \\
Solution 2 & $12.7047 \pm 0.0011$ & $0.076 \pm 0.003$ \\
\hline
\end{tabular}

Note: the two solutions could also be considered as extremes of a broader range of possible, although less likely, rotational periods.

uncertainty on the rotational period $T_{\text {rot }}$ is below $5 \mathrm{~s}$, and the error on the linear phase function coefficient $\beta$ is $0.003 \mathrm{mag} /{ }^{\circ}$. The results are summarised in Table 2.

Application of a similar analysis to the $67 \mathrm{P} / \mathrm{C}-\mathrm{G}$ data, now using the IAU-adopted phase law with slope parameter $G$, provides the following results: both the PDM and the $\chi^{2}$ minimisation methods have a global minimum of $\Theta$ at the rotational frequency $f_{3}$ identical (within its error) to the solution $f_{2}$ determined before for the linear phase law. The second deepest minima obtained with both methods differ from $f_{1}$ and also from each other. This suggests that the solution $f_{2}\left(\equiv f_{3}\right)$ is very robust, because insensitive to the mathematical form of the phase function. In contrast, solution $f_{1}$ is not very stable in this respect.

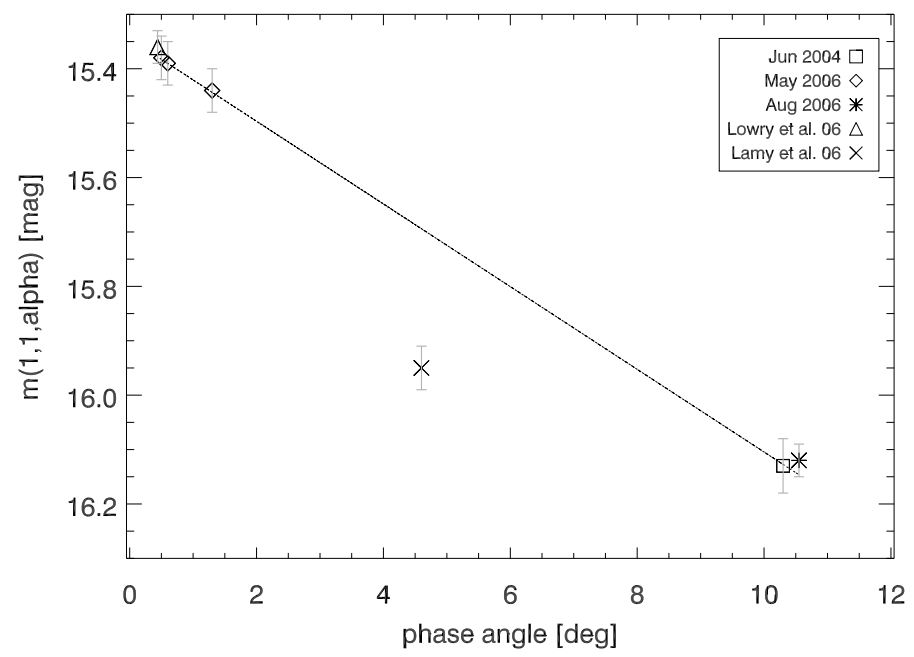

Fig. 5. Reduced magnitude vs. phase angle. The values correspond to the midpoint magnitude in $R$ filter. The different symbols correspond to the different datasets. The solid line indicates the slope of $\beta=0.076 \mathrm{mag} /{ }^{\circ}$.

This global minimum is found at $G$ equal to $-0.08 \pm 0.02$ (for PDM) and $-0.09 \pm 0.02$ (for the $\chi^{2}$ minimisation method), practically identical values, which corresponds to a shallower brightness dependence on the phase angle than the one derived for this comet by Lamy et al. (2007), who obtained $G=-0.45$.

Discussion. We consider the analysis employing a linear phase function approximation to be more realistic, since the quality of the profiles, indicated by the $\Theta$ parameter, is better than for the IAU-adopted approximation. This could be interpreted that for $67 / \mathrm{C}-\mathrm{G}$, the opposition effect is rather not very pronounced. The two solutions have the same statistical significance, and offer practically the same rotation phase profiles. However, solution 2 is additionally supported by the analysis with the alternative phase function.

Figure 5 shows the behaviour of the reduced magnitude $m(1,1, \alpha)$ vs. phase angle for each dataset. Since in August 2006 the phase angle is almost constant during the nights of observation, one single value has been calculated for that observing run. The solid line corresponds to a linear phase coefficient $\beta=0.076 \mathrm{mag} /{ }^{\circ}$. For comparison the midpoint reduced magnitude from Lowry et al. (2006) and Lamy et al. (2006) are overplotted. While the value from Lowry et al. (2006) is in excellent agreement with ours, the midpoint reduced magnitude from Lamy et al. (2006) is much lower. This can explain the very steep phase dependence for this comet obtained by Lamy et al. (2007), who used data only from Lowry et al. (2006) and Lamy et al. (2006) to determine the slope parameter $G$. Figure 5 also suggests that the measurement by Lamy et al. (2006) underestimates the brightness of the comet.

Using the value for the linear phase coefficient $\beta_{2}=0.076 \pm$ $0.003 \mathrm{mag} /{ }^{\circ}$, we determined the absolute magnitudes for the June 2004 run. The partial light curve from June 2004 matches the phase profile obtained from the May and August 2006 observations well, when arbitrarily shifted in rotational phase to match best (estimated by eye) the 2006 rotation light curve (Fig. 6).

Our best solution for the rotational period of the nucleus $\left(T_{\text {rot }, 2}=12.7047 \pm 0.0011 \mathrm{~h}\right)$ is in excellent agreement with the period published by Lowry et al. (2006), who found 12.72 \pm $0.05 \mathrm{~h}$. However, it is more than one order of magnitude more accurate than the latter. Also our second solution $T_{\text {rot }, 1}$ is close 


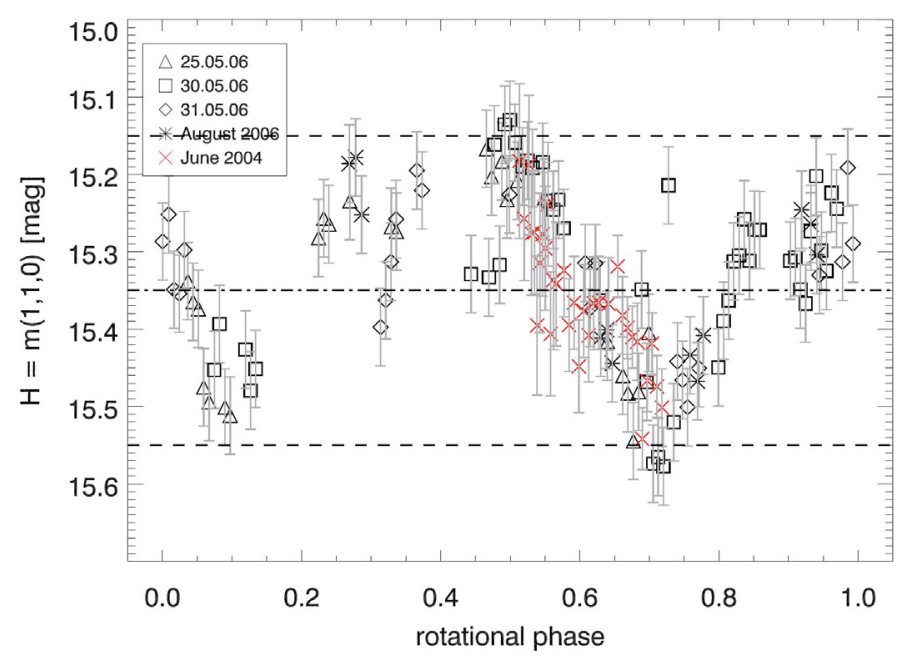

Fig. 6. Phased light curve of 67P/C-G from May \& August 2006 datasets, overplotted with the observations of June 2004, arbitrarily located in rotational phase.

to this value. The phase profiles from both studies are doublepeaked, with the same amplitude $\Delta m$. This is compatible with a light curve dominated by the shape of the nucleus. Comparing the phase profile of Lowry et al. (2006) with ours, both phased light curves show the secondary peak to be a bit fainter with respect to the primary one. In addition, the main peak presents a modulation in our phase profile, which was not found by Lowry et al. (2006). The rotational period $T_{\text {rot }}=12.41 \pm 0.41 \mathrm{~h}$, published by Lamy et al. (2006), is consistent with our result within its error, although it is less accurate by more than two orders of magnitude. By applying our methods to the HST nucleus photometry of $67 \mathrm{P} / \mathrm{C}-\mathrm{G}$ by Lamy et al. (2006), we find plausible solutions for rotational periods of $6.1 \mathrm{~h}, 12.7 \mathrm{~h}, 16.1 \mathrm{~h}$, and $18.4 \mathrm{~h}$, where the solution at $T_{\text {rot }}=12.7 \pm 0.2 \mathrm{~h}$, corresponding to the one they published, is now perfectly consistent with our result.

\subsection{Size and shape of the nucleus}

The rotational phase profile has an asymmetric, double-peaked profile, with a maximum amplitude $\Delta m=0.40 \pm 0.07 \mathrm{mag}$. Assuming an ellipsoidal shape for the nucleus, with semi-major axes $a$ and $b$ and constant surface albedo, we can estimate a lower limit for the axis ratio using

$\frac{a}{b} \geq 10^{(0.4 \cdot \Delta m)}=1.45 \pm 0.09$.

To obtain the absolute magnitude $H=m(1,1,0)$, we have to apply a phase correction to the reduced magnitude $m(1,1, \alpha)$. We consider a linear phase law (similar to Lamy et al. 2004) with $\beta=0.076 \pm 0.003 \mathrm{mag} /{ }^{\circ}$, as determined in Sect. 3.2 and calculate the effective radius of the nucleus of $67 \mathrm{P} / \mathrm{C}-\mathrm{G}$ using

$R_{\mathrm{eff}}=\left[\frac{10^{0.4\left[M_{\odot}-H\right]}}{A}\right]^{1 / 2}$,

where $M_{\odot}$ is the absolute magnitude of the Sun in the $R$ filter, $H$ the mean absolute magnitude of the comet, and $A$ its albedo. We obtained an effective nucleus radius $R_{\text {eff }}=2.38 \pm 0.04 \mathrm{~km}$, assuming an albedo of 0.04 .

Discussion. The mean absolute $R$ filter magnitude of the comet ( $H=15.35 \pm 0.04 \mathrm{mag})$ is consistent with the one obtained
Table 3. Colour indices of $67 \mathrm{P} / \mathrm{C}-\mathrm{G}$ as determined from the photometric observations in June 2004 and May 2006.

\begin{tabular}{cccc}
\hline \hline & $V-R(\mathrm{mag})$ & $R-I(\mathrm{mag})$ & $\phi^{\star}$ \\
\hline 16.06 .2004 & $0.38 \pm 0.04$ & $0.44 \pm 0.06$ & \\
\hline 25.05 .2006 & $0.49 \pm 0.06$ & - & 0.1 \\
& $0.65 \pm 0.07$ & $0.45 \pm 0.04$ & 0.3 \\
& $0.66 \pm 0.08$ & $0.47 \pm 0.05$ & 0.5 \\
\hline 30.05 .2006 & - & $0.40 \pm 0.09$ & 0.6 \\
& $0.55 \pm 0.08$ & $0.39 \pm 0.07$ & 0.9 \\
\hline 31.05 .2006 & $0.44 \pm 0.08$ & - & 0.4 \\
& $0.46 \pm 0.07$ & $0.54 \pm 0.06$ & 0.8 \\
& $0.50 \pm 0.06$ & $0.57 \pm 0.06$ & 0.9 \\
\hline
\end{tabular}

Note: ${ }^{\star}$ the rotational phase at which $V$ and $I$ filter images, used to determine the corresponding colour indices, were taken.

by Lowry et al. (2006). 67P/C-G appears slightly brighter than expected from the HST photometry by Lamy et al. (2006), supporting the interpretation as being due to a changing viewing geometry of the nucleus along the orbit. Our radius value is higher than the one determined by Lamy et al. (2006) and Lowry et al. (2006). This discrepancy is due to them using a linear phase coefficient of $0.04 \mathrm{mag} /{ }^{\circ}$. On the other hand, our result can be potentially biased by the opposition effect of the nucleus, which may result in overestimating the nucleus size. Up to now no proof of the presence of the opposition effect for comet $67 \mathrm{P} / \mathrm{C}-\mathrm{G}$ exists. For a shallower linear phase function coefficient of $0.04 \mathrm{mag} /{ }^{\circ}$, we obtain an effective radius of the nucleus of $2.00 \mathrm{~km}$. Our result for the axis ratio $(a / b \geq 1.45 \pm 0.09)$ is in good agreement with the one obtained by Lowry et al. (2006).

\subsection{Surface colours and reflectance spectra of the nucleus}

The colour indices $(V-R)$ and $(R-I)$ were obtained from the June 2004 and May 2006 observations (see Table 3). They show that the comet's nucleus is slightly redder than the Sun $\left((V-R)_{\odot}=0.36 \mathrm{mag},(R-I)_{\odot}=0.33 \mathrm{mag}\right)$, as expected for cometary nuclei (Lamy et al. 2004). The August 2006 observations were only performed in $R$ filter, thus the determination of colour indices is not possible.

In May 2006, $V$ and $I$ filter images of the comet were obtained at different rotational phases (Table 3 ). Thus, the resulting colour indices sample different regions of the nucleus surface.

The values for $R-I$ index are rather uniform. The $V-R$ indices, in particular on May 25, show a larger scatter, in parts due to having too few measurements performed in $V$ filter. In fact, only one single $V$ filter image was taken per epoch. Although slightly outside the error bars of the other $V-R$ results, the higher $V-R$ colours are still consistent with the measured spectra of the cometary nucleus (see Fig. 7). For each couple of $V-R$ and $R-I$ colours in Table 3, we determined the mean spectral reddening and found it to be constant within the error bars. Thus, we conclude that colour variations with the rotational phase are small, if present at all. The average $V-R$ and $R-I$ colours of the nucleus of $67 \mathrm{P} / \mathrm{C}-\mathrm{G}$ are found to be $(0.54 \pm 0.03) \mathrm{mag}$ and $(0.47 \pm 0.03) \mathrm{mag}$, respectively, and compare to a mean spectral reddening (Delsanti et al. 2001) of $(11 \pm 2) \% / 1000 \AA$.

Visible spectra of the comet were observed on May 25 and May 31, 2006. Two spectra were discarded because of unreasonable spectral shapes possibly caused by a background object passing across the comet image while integrating the spectrum. Each spectrum was binned to $70 \AA$ A/pix. Figure 7 shows 


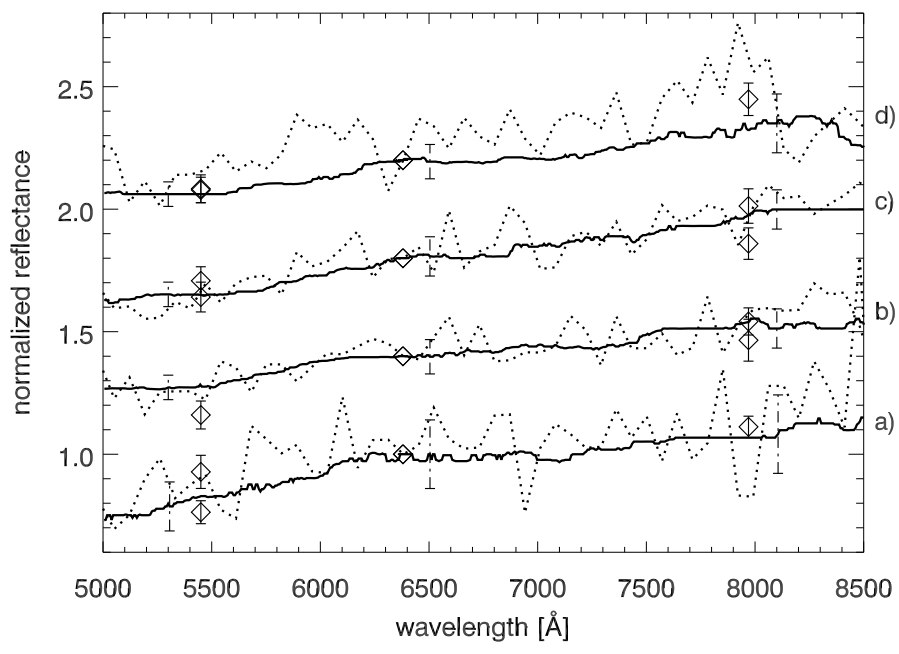

Fig. 7. Spectra of the comet $67 \mathrm{P} / \mathrm{C}-\mathrm{G}$. To each binned spectrum (dotted line) the corresponding smoothed one (solid line) is superimposed. Spectrum a) was observed on May 25, while b), c), and d) were taken on May 31, 2006. The spectra are normalised to unity at $6380 \AA$ and shifted for clarity. The diamonds superimposed to the spectra correspond to the spectral reflectivities obtained from the photometric colour indices of the nucleus, closest in rotational phase. The dashed bars beside the diamonds show the uncertainties of the binned reflectance spectra in each wavelength region, as summarised in Table 4.

Table 4. Signal-to-noise ratio $(S / N)$ and corresponding relative error for the binned spectra shown in Fig. 7.

\begin{tabular}{cccc}
\hline \hline Spectrum & $\lambda^{\star}(\AA)$ & $S / N$ & error \\
\hline $\mathrm{a}$ & $5000-5140$ & 10 & 0.10 \\
& $6500-6640$ & 7 & 0.14 \\
& $8000-8140$ & 6 & 0.16 \\
\hline $\mathrm{b}$ & $5000-5140$ & 21 & 0.05 \\
& $6500-6640$ & 14 & 0.07 \\
& $8000-8140$ & 13 & 0.08 \\
\hline $\mathrm{c}$ & $5000-5140$ & 19 & 0.05 \\
& $6500-6640$ & 13 & 0.08 \\
& $8000-8140$ & 13 & 0.08 \\
\hline $\mathrm{d}$ & $5000-5140$ & 19 & 0.05 \\
& $6500-6640$ & 15 & 0.07 \\
& $8000-8140$ & 8 & 0.12 \\
\hline
\end{tabular}

Note: ${ }^{\star}$ the wavelength range used for the determination of $S / N$ and the error.

the binned spectra of $67 \mathrm{P} / \mathrm{C}-\mathrm{G}$ with the smoothed ones superimposed.

Spectra a) and b) have been observed at rotational phase between 0.4 and 0.5 , while c) and d) at rotational phase between 0.8 and 0.95 . The $S / N$ ratios and the corresponding relative errors of the individual binned spectra are summarised in Table 4.

The spectra display a reddening of rather constant slope of $\sim 10 \% / 1000 \AA$ and do not show any absorption or emission features. The reddening obtained from the spectra agrees within the errors with the one calculated from the photometric colours of the nucleus (see Fig. 7). Constant reddening of the reflectance spectra with rotational phase also agrees with the previous conclusion of no (or only small) photometric colour variation of the nucleus with rotational phase. Thus, we conclude that the nucleus colour and/or reflectance does not vary much over rotational phase.

Discussion. Lowry et al. (2006) obtained a colour index ( $V-$ $R)=0.41 \pm 0.04 \mathrm{mag}$, in good agreement with our results.

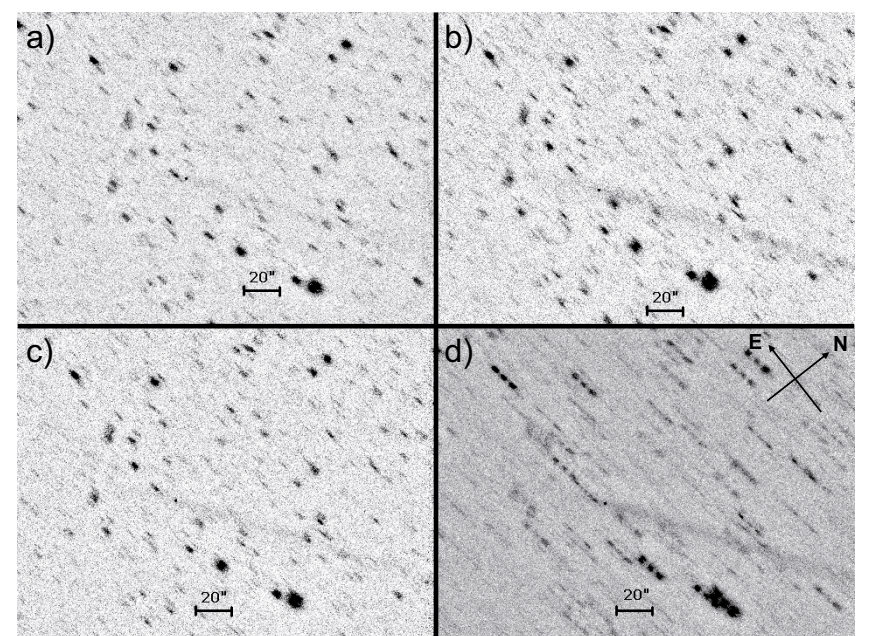

Fig. 8. Trail-like structure of $67 \mathrm{P} / \mathrm{C}-\mathrm{G}$ in a) $I$ filter, b) $R$ filter, c) $V$ filter, d) composite VRI image. All images are centred on the nucleus of the comet (black spot close to the panel centres). All subpanels have the same orientation, which is indicated in panel d). The trail-like structure has an extension of more than $153.25^{\prime \prime}$ and a width of about $8^{\prime \prime}$, respectively $\sim 4.8 \times 10^{5} \mathrm{~km}$ and $\sim 2.5 \times 10^{4} \mathrm{~km}$ at the comet distance.

Lamy et al. (2006) claim a variation in the nucleus colour across the surface of the nucleus, a result that is not confirmed by our photometry and spectroscopy. Their averaged $(V-R)$ colour index agrees with our results, while they obtain a photometric reflectivity gradient of $17 \% / 1000 \AA$, slightly higher than the reflectivity slope we obtained from our spectra. Since reflectance spectra provide a more detailed picture of the reddening behaviour, we favour our conclusion of rather uniform surface reflectance and colours of the nucleus of comet $67 \mathrm{P} / \mathrm{C}-\mathrm{G}$. The absence of gas emission features in the spectra is not surprising, since the comet is found without coma, as described above.

\subsection{Dust trail}

Dust trails are commonly associated with short-period comets (Sykes et al. 1986). They are usually formed by mm-sized and larger particles and appear to the observers as faint and linear structures. The dust trail of $67 \mathrm{P} / \mathrm{C}-\mathrm{G}$ was observed for the first time in the IR by the Infrared Astronomical Satellite (IRAS) in 1983 (Agarwal et al. 2007, and references therein) .

Our June 2004 observations were dedicated to the detection and characterisation of the dust trail in the visible wavelength range. Due to its low $S / N$ ratio, it cannot be detected using a single exposure of the comet field. To allow detection, a median combination of the images (filter by filter) centred on the comet was applied. Figure 8 shows the result of the median combination in $I, R$, and $V$ filters and the composite image, obtained as the average of the three filter exposures.

A trail-like structure is clearly visible in the mediancombined images, projected in the sunward direction, at a position angle $\mathrm{PA}=(298.1 \pm 0.2)^{\circ}$, measured north over east. This value agrees more with the predicted position angle of the neck-line of $(298.4 \pm 0.2)^{\circ}$ (Agarwal, private communication), rather than the heliocentric velocity vector projected in the sky $\left(297.2^{\circ}\right)$, which indicated the orientation of the dust trail. Thus, based only on geometrical considerations, the trail-like structure is better interpreted as a neck-line rather than as a trail. At a distance of $\sim 47^{\prime \prime}\left(\sim 1.5 \times 10^{5} \mathrm{~km}\right.$ at the distance of the comet $)$ from the nucleus, we measured a surface brightness of the trail-like 
Table 5. Results of photometry in $R$ filter.

\begin{tabular}{|c|c|c|c|c|c|c|c|c|c|c|c|}
\hline Time (h) & $m(1,1, \alpha)$ & $\sigma$ & Day & Time (h) & $m(1,1, \alpha)$ & $\sigma$ & Day & Time (h) & $m(1,1, \alpha)$ & $\sigma$ & Day \\
\hline 0.465 & 15.44 & 0.05 & 1 & 124.496 & 15.49 & 0.05 & 2 & 2017.112 & 16.26 & 0.05 & 4 \\
\hline 0.560 & 15.46 & 0.05 & 1 & 124.590 & 15.43 & 0.05 & 2 & 2017.220 & 16.20 & 0.05 & 4 \\
\hline 0.657 & 15.47 & 0.05 & 1 & 124.683 & 15.41 & 0.05 & 2 & 2040.754 & 16.21 & 0.05 & 5 \\
\hline 0.751 & 15.57 & 0.05 & 1 & 124.777 & 15.35 & 0.05 & 2 & 2040.863 & 16.20 & 0.05 & 5 \\
\hline 0.854 & 15.59 & 0.05 & 1 & 124.875 & 15.35 & 0.05 & 2 & 2040.971 & 16.24 & 0.05 & 5 \\
\hline 1.143 & 15.60 & 0.05 & 1 & 124.969 & 15.30 & 0.05 & 2 & 2137.801 & 15.99 & 0.05 & 6 \\
\hline 1.237 & 15.61 & 0.05 & 1 & 125.063 & 15.35 & 0.05 & 2 & 2137.918 & 15.98 & 0.05 & 6 \\
\hline 2.850 & 15.38 & 0.05 & 1 & 125.156 & 15.31 & 0.05 & 2 & 2138.027 & 16.06 & 0.05 & 6 \\
\hline 2.944 & 15.35 & 0.05 & 1 & 125.250 & 15.31 & 0.05 & 2 & 2158.769 & 16.05 & 0.05 & 7 \\
\hline 3.040 & 15.36 & 0.05 & 1 & 125.809 & 15.35 & 0.05 & 2 & 2158.937 & 16.07 & 0.05 & 7 \\
\hline 3.421 & 15.33 & 0.05 & 1 & 125.902 & 15.35 & 0.05 & 2 & 2159.046 & 16.11 & 0.05 & 7 \\
\hline 4.179 & 15.37 & 0.05 & 1 & 125.996 & 15.39 & 0.05 & 2 & -0.340 & 15.97 & 0.10 & 8 \\
\hline 4.274 & 15.37 & 0.05 & 1 & 126.089 & 15.41 & 0.05 & 2 & -0.252 & 16.04 & 0.08 & 8 \\
\hline 5.918 & 15.26 & 0.05 & 1 & 126.184 & 15.32 & 0.05 & 2 & -0.168 & 15.97 & 0.09 & 8 \\
\hline 6.013 & 15.30 & 0.05 & 1 & 126.282 & 15.24 & 0.05 & 2 & -0.117 & 16.06 & 0.08 & 8 \\
\hline 6.201 & 15.28 & 0.05 & 1 & 126.376 & 15.34 & 0.05 & 2 & -0.065 & 16.06 & 0.08 & 8 \\
\hline 6.295 & 15.33 & 0.05 & 1 & 126.470 & 15.37 & 0.05 & 2 & -0.016 & 16.18 & 0.09 & 8 \\
\hline 6.488 & 15.31 & 0.05 & 1 & 126.563 & 15.27 & 0.05 & 2 & 0.033 & 16.10 & 0.09 & 8 \\
\hline 6.980 & 15.33 & 0.05 & 1 & 126.657 & 15.29 & 0.05 & 2 & 0.082 & 16.06 & 0.08 & 8 \\
\hline 8.126 & 15.51 & 0.05 & 1 & 127.994 & 15.49 & 0.05 & 2 & 0.132 & 16.08 & 0.08 & 8 \\
\hline 8.408 & 15.56 & 0.05 & 1 & 128.088 & 15.44 & 0.05 & 2 & 0.182 & 16.02 & 0.08 & 8 \\
\hline 8.503 & 15.58 & 0.05 & 1 & 128.562 & 15.47 & 0.05 & 2 & 0.231 & 16.19 & 0.08 & 8 \\
\hline 8.602 & 15.64 & 0.05 & 1 & 128.656 & 15.52 & 0.05 & 2 & 0.280 & 16.12 & 0.09 & 8 \\
\hline 8.695 & 15.58 & 0.05 & 1 & 128.750 & 15.49 & 0.05 & 2 & 0.329 & 16.13 & 0.08 & 8 \\
\hline 8.884 & 15.50 & 0.05 & 1 & 143.737 & 15.44 & 0.05 & 3 & 0.476 & 16.11 & 0.06 & 8 \\
\hline 119.979 & 15.37 & 0.05 & 2 & 143.831 & 15.40 & 0.05 & 3 & 0.566 & 16.18 & 0.06 & 8 \\
\hline 120.305 & 15.38 & 0.05 & 2 & 143.925 & 15.35 & 0.05 & 3 & 0.655 & 16.15 & 0.06 & 8 \\
\hline 120.405 & 15.20 & 0.05 & 2 & 144.019 & 15.30 & 0.05 & 3 & 0.746 & 16.23 & 0.06 & 8 \\
\hline 120.502 & 15.36 & 0.05 & 2 & 144.398 & 15.24 & 0.05 & 3 & 0.835 & 16.16 & 0.06 & 8 \\
\hline 120.595 & 15.18 & 0.05 & 2 & 144.492 & 15.26 & 0.05 & 3 & 0.925 & 16.19 & 0.06 & 8 \\
\hline 120.689 & 15.17 & 0.05 & 2 & 146.089 & 15.27 & 0.05 & 3 & 1.015 & 16.15 & 0.06 & 8 \\
\hline 120.782 & 15.20 & 0.05 & 2 & 147.472 & 15.35 & 0.05 & 3 & 1.105 & 16.15 & 0.06 & 8 \\
\hline 120.915 & 15.23 & 0.05 & 2 & 147.566 & 15.41 & 0.05 & 3 & 1.285 & 16.15 & 0.06 & 8 \\
\hline 121.009 & 15.22 & 0.05 & 2 & 147.660 & 15.36 & 0.05 & 3 & 1.453 & 16.10 & 0.04 & 8 \\
\hline 121.102 & 15.23 & 0.05 & 2 & 149.157 & 15.48 & 0.05 & 3 & 1.543 & 16.17 & 0.05 & 8 \\
\hline 121.289 & 15.23 & 0.05 & 2 & 149.251 & 15.51 & 0.05 & 3 & 1.633 & 16.18 & 0.04 & 8 \\
\hline 121.387 & 15.28 & 0.05 & 2 & 149.344 & 15.54 & 0.05 & 3 & 1.722 & 16.20 & 0.04 & 8 \\
\hline 121.481 & 15.29 & 0.05 & 2 & 149.550 & 15.49 & 0.05 & 3 & 1.813 & 16.20 & 0.05 & 8 \\
\hline 121.575 & 15.27 & 0.05 & 2 & 151.751 & 15.37 & 0.05 & 3 & 1.903 & 16.33 & 0.04 & 8 \\
\hline 121.669 & 15.31 & 0.05 & 2 & 152.172 & 15.35 & 0.05 & 3 & 1.993 & 16.25 & 0.04 & 8 \\
\hline 122.338 & 15.41 & 0.05 & 2 & 152.270 & 15.23 & 0.05 & 3 & 2.083 & 16.20 & 0.04 & 8 \\
\hline 123.094 & 15.39 & 0.05 & 2 & 152.365 & 15.33 & 0.05 & 3 & 2.172 & 16.26 & 0.04 & 8 \\
\hline 123.188 & 15.51 & 0.05 & 2 & 152.459 & 15.33 & 0.05 & 3 & 2.263 & 16.29 & 0.05 & 8 \\
\hline 123.304 & 15.62 & 0.05 & 2 & 152.570 & 15.29 & 0.05 & 3 & & & & \\
\hline 123.398 & 15.61 & 0.05 & 2 & 152.669 & 15.39 & 0.05 & 3 & & & & \\
\hline 123.491 & 15.62 & 0.05 & 2 & 152.763 & 15.39 & 0.05 & 3 & & & & \\
\hline 123.586 & 15.26 & 0.05 & 2 & 152.857 & 15.34 & 0.05 & 3 & & & & \\
\hline 123.680 & 15.56 & 0.05 & 2 & 2016.982 & 16.23 & 0.05 & 4 & & & & \\
\hline
\end{tabular}

Explanation: $m(1,1, \alpha)$ is the reduced magnitude and $\sigma$ its error. Day is the day of the observation $(1=$ May 25,$2006 ; 2=$ May 30,$2006 ; 3=$ May 31, 2006; 4 = August 17, 2006; 5 = August 18, 2006; 6 = August 22, 2006; 7 = August 23, 2006; 8 = June 16, 2004). The time relative to May and August 2006 runs is normalised to May 26, 2006 00:00 UT. The time series of June 2004, instead, is normalised to June 16, 2004 23:20 UT. The times are light-time corrected.

structure of $(28.1 \pm 0.1) \mathrm{mag} /{ }^{\prime \prime 2}$ in $R$ filter, $(29.0 \pm 0.1) \mathrm{mag} /{ }^{\prime \prime 2}$ in $V$ filter, and $(27.7 \pm 0.1) \mathrm{mag} /{ }^{\prime \prime 2}$ in $I$ filter. Thus we determined for the first time its visible colours: $(V-R)=0.95 \pm 0.14 \mathrm{mag} /{ }^{\prime \prime 2}$ and $(R-I)=0.39 \pm 0.14 \mathrm{mag} /{ }^{\prime \prime 2}$. The corresponding reflectance presents a steeper increase between $5500 \AA$ and $6400 \AA$, while it becomes much shallower between $6400 \AA$ and $8000 \AA$. Over the whole wavelength range (5500-8000 $\AA$ ), we obtained a mean spectral slope of $\sim 17 \% / 1000 \AA$. Thus, on average, the traillike structure looks redder than the nucleus of $67 \mathrm{P} / \mathrm{C}-\mathrm{G}$, which presents a reddening slope of $\sim 10 \% / 1000 \AA$.
In a similar way, we tried to detect a trail-like structure also in the $R$-band images taken in May 2006. As previously discussed, the field of view was very crowded from bright stars from the galactic disc. Even applying a median combination of the images centred in the comet, "remanence" effects from the background objects produce artificial linear structures in the median-combined image, as can be seen in Fig. 9. However, a faint structure is visible in the expected direction $(\mathrm{PA}=$ $(285.6 \pm 0.2)^{\circ}$ measured north over east), but no quantitative characterisation of the trail-like structure can be done. 


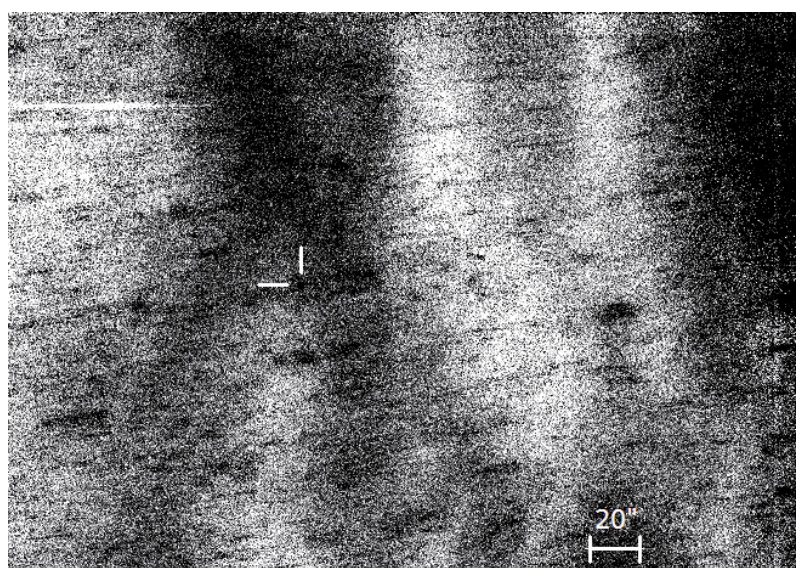

Fig. 9. Median combination of $R$ filter images taken on May 31, 2006. The orientation of the image is north up, east left. The nucleus of the comet is the black dot close to the centre of the image. A faint structure at $P A=(285.6 \pm 0.2)^{\circ}$ is visible. The image quality is very poor. In fact, due to the crowdiness of the field, the median combination technique did not work well in removing background objects. Their "remanences" produce artificial linear structures in the median-combined image.

\section{Summary and conclusions}

We analysed broad-band images and low-resolution spectra obtained at the ESO VLT in June 2004, May 2006, and August 2006, when 67P/C-G was at the heliocentric distance of $r \geq$ 4.9 AU. At all epochs the comet looks point-like, which indicates the absence (or very low level) of coma activity around the nucleus.

Using the PDM and the $\chi^{2}$ minimisation methods, we determined that the best solution for the rotational period of the comet is $T_{\text {rot }}=12.7047 \pm 0.0011 \mathrm{~h}$. A second solution is visible in the periodograms $\left(T_{\text {rot }}=12.7889 \pm 0.0009 \mathrm{~h}\right)$, which looks less stable and robust than the previous one. These two solutions could also be considered as the extremes of a broad range of possible, although less likely, rotational periods. The bulk of the nuclei of ecliptic comets have rotational periods between 5 and $18 \mathrm{~h}$ (Lamy et al. 2004), the rotational period we determined for the nucleus of $67 \mathrm{P} / \mathrm{C}-\mathrm{G}$ is in the afore-mentioned range. No evidence of a pronounced opposition effect is present in the data, in particular not for the May 2006 observations that cover the phase angle range of $0.5-1.3^{\circ}$. The linear approximation of the phase function gives a better quality to the rotational phase profile than the IAU-adopted law. We estimated a linear phase coefficient $\beta=0.076 \pm 0.003 \mathrm{mag} /{ }^{\circ}$, higher than the one usually adopted for cometary nuclei (Lamy et al. 2004). From the rotational phase profile, we calculated a lower limit for the axis ratio of $1.45 \pm 0.09$ and an effective radius of the nucleus of $2.38 \pm 0.04 \mathrm{~km}$, assuming an albedo of 0.04 and the linear phase coefficient previously determined. Both these results are in the range of values determined for ecliptic comets (Lamy et al. 2004). From both photometry and spectroscopy, the nucleus of $67 \mathrm{P} / \mathrm{C}-\mathrm{G}$ looks slightly redder than the Sun, and does so uniformly over the nuclear surface, with a reflectivity gradient of $\sim 10 \% / 1000 \AA$. Also in this respect, $67 \mathrm{P} / \mathrm{C}-\mathrm{G}$ behaves as expected for Jupiter family comets (Lamy et al. 2004). A trail-like structure is visible in the June 2004 observation with an average brightness in the $\mathrm{R}$ filter of $\sim 28 \mathrm{mag} /{ }^{\prime \prime 2}$. We determined its visible colours for the first time: $(V-R)=0.95 \pm 0.14 \mathrm{mag} /{ }^{\prime \prime 2}$ and $(R-I)=0.39 \pm 0.14 \mathrm{mag} /{ }^{\prime \prime 2}$. The trail-like structure is barely visible in the May 2006 images.

Acknowledgements. Thanks go to Gian Paolo Tozzi (INAF - Oss. Astronomico di Arcetri, Italy), who gave precious help in the reduction and extraction of the spectra, and to Wacław Waniak (Astronomical Observatory, Jagiellonian University, Kraków, Poland), who co-developed some software used for this work. We would like to thank the astronomers and operation team of the VLT observatory for their skilled support during the visitor mode observations and for their careful work for our programs in service mode. We also thank Rita Schulz (ESTEC/ESA), Gerhard Schwehm (ESTEC/ESA), Jogy Stuewe (Leiden Observatory), and Jose-Luis Ortiz (IAA Granada, Spain) for their support for the proposals.

\section{References}

Agarwal, J., Mueller, M, \& Gruen, E. 2007, Space Sci. Rev., 128, 79 Boehnhardt, H., Delahodde, C., Sekiguchi, T., et al. 2002, A\&A, 387, 11071113.

Bowell, E., Hapke, B., Domingue, D., \& Harris, A. W. 1989, in Asteroids II, ed. R. P. Binzel, T. Gehrels, \& M. S. Matthews (University of Arizona Press), 524

Delsanti, A. C., Boehnhardt, H., Barrera, L., et al. 2001, A\&A, 380, 347

Drahus, M., \& Waniak, W. 2006, Icarus, 185, 544

Fernandez, Y. R., Lisse, C. M., Ulrich Kaeufl, H., et al. 2000, Icarus, 147, 145 Hansen, K. C., Bagdonat, T., Motschmann, U., et al. 2007, Space Sci. Rev., 128, 133

Lamy, P. L., Toth, I., Fernandez, Y. R., \& Weaver, H. A. 2004, in Comets II, ed. M. C. Festou, H. U. Keller, \& H. A. Weaver (Tucson: University of Arizona Press), 223

Lamy, P. L., Toth, I., Weaver H. A., et al. 2006, A\&A, 458, 669

Lamy, P. L., Toth, I., Davidsson, B. J. R., et al. 2007, Space Sci. Rev., 128, 23

Lowry, S. C., Fitzsimmons, A., Jorda, L., et al. 2006, DPS meeting \#38, \#08.01; BAAS, 38, 492

Meeus, J. 1998, in Astronomical algorithms, 2nd edn., ed. J. Meeus (Richmond, VA: Willmann-Bell)

Michałowski, T. 1988, Acta Astron., 38, 455

Stellingwerf, R. F. 1978, ApJ, 224, 953

Sykes, M. V., Lebofsky, L. A., Hunten, D. M., \& Low, F. 1986, Science, 232, 1115 